\title{
Oral manifestations and dental management of patient with leukocyte alterations
}

\author{
Aisha Mancheño Franch ${ }^{1}$, Carmen Gavaldá Esteve², M Gracia Sarrión Pérez $^{1}$ \\ ${ }^{1}$ Dentist. \\ ${ }^{2}$ Staff physician, Service of Stomatology, Valencia University General Hospital.
}

Correspondence:

C/ Prof. Dr. Severo Ochoa 4, esc. izda. pta. 4

46010 Valencia

E-mail: aishamancheno@gmail.com

\begin{abstract}
Leukocytes are the main cellular elements of inflammatory and immune reactions of the organism. Leukocyte alterations are the consequence of an imbalance between the formation of leukocytes in the bone marrow and its elimination by the mononuclear phagocytic system. Factors that can modify leukopoyesis are varied and can lead to an alteration in the number of leukocytes or tumoral alterations of white cells (leukemias, lymphomas and plasma cell tumors). There is also a wide range of clinical manifestations that can derive from them: from very slight symptoms to life-threatening conditions. In some cases oral manifestations will be the first signs and it will be the dentist's responsibility to identify the underlying disorder and guide the diagnosis of the patient. It is important to be familiar with the special management required for these patients, in which the dental treatment can affect the course of the underlying disease. The objective of this article is to review the literature concerning the oral manifestations and the considerations that must factor in the dental treatment of patients with leukocyte alterations.
\end{abstract}

Key words: leukocyte alterations, oral manifestations, dental management. 


\section{Introduction}

Leukocytes are formed in the bone marrow and then transported by the blood to different tissues. Normal counts of leukocytes in blood are 4.500-10.000 cells/ $\mathrm{mm}^{3}$.

Leukocyte alterations are the consequence of an imbalance between their formation in the bone marrow and subsequent elimination by the mononuclear phagocytic system.

Factors that can modify leukopoyesis are varied and can lead to an alteration in the number of leukocytes or tumoral alterations of white cells (leukemias, lymphomas and plasma cell tumors). There is also a wide range of clinical manifestations that can derive from them: from very slight symptoms to life threatening conditions. In some cases oral manifestations will be the first signs and it will be responsibility of the dentist to identify that an underlying disorder is present and to guide the diagnosis of the patient; in the case of patients already diagnosed, special considerations will be required to manage these patients, in which the dental treatment can affect the course of the underlying disease.

\section{Objectives}

The aim of this article is to carry out a review of literature about leukocyte alterations, and to examine the latest research concerning the oral manifestations and dental management of patients with the associated conditions.

\section{Material and Methods}

The subject-related research was carried out on the Pubmed-Medline database. As a research strategy we employed different combinations of the following key words: oral complications, oral manifestations, dental management, agranulocytosis, neutropenia, leukocytosis, hematologic malignancies, lymphoma, leukemia, mieloma.

We included articles published in both English and Spanish, dating from 1995. We reviewed 21 articles.

\section{Results}

\section{ALTERATIONS IN THE NUMBER OR FUNCTION} OF LEUCOCYTES

\section{Leukopenia}

Leukopenia is a decrease in the total number of leukocytes under $4.000-4.500 / \mathrm{mm}^{3}$. However, even though leukopenias can be associated with a global decrease in leukocytes, they are usually caused by an impaired capacity to generate neutrophils.

Neutropenia is defined as a total circulating neutrophil count lower than $2000 \times 10^{6} /$ litre (1). It is not a disease but a sign of an underlying disorder with a wide range of underlying causes. Decreased production of neutrophils is associated with deficiencies of vitamin B12 and folic acid, aplastic anemia, tumors (leukemia, solid tu- mors that invade bone marrow), drugs, metabolic diseases (glycogen storage disease type 1b, methylomalonic acidaemia), nutritional deficiencies (malnutrition,copper deficiency), radiation injury, immune mechanisms, and heritable genetic aberrations (congenital neutropenia, cyclic neutropenia). Certain infections decrease the number of neutrophils in the circulating blood due to increased migration of neutrophils into the tissue, sequestration of neutrophils, or direct toxic effect of the microorganism and its toxins on the bone marrow (2).

\section{Neutropenias that can cause oral manifestations.}

In general, the most common oral complications related to neutropenia are ulcers, severe gingivitis and periodontitis (1).

Acquired agranulocytosis or neutropenia is far more common than congenital forms, often accompanying viral infections, or it may be attributable to drugs in $>$ $70 \%$ of cases, which is a rare and potentially life-threatening condition, owing to either myelosuppression or antibody-mediated destruction. Drugs associated with agranulocytosis include antiepileptics, antithyroid drugs (carbimazole, methimazole, and propylthiouracil), antibiotics (penicillin, chloramphenicol, and cotrimoxazole), cytotoxic drugs, gold, nonsteroidal antiinflammatory drugs (indomethacin, naproxen, and phenylbutazone), mebendazole, the antidepressant mirtazapine, and some antipsychotics (clozapine) (2). Also metamizole, a drug frequently prescribed by dentists, may cause agranulocytosis (3).

In the oral cavity, agranulocytosis can appear as necrotic ulcers with a white or greyish surface without signs of inflammation $(2,3)$.

The mortality rate for agranulocytosis is 7 to $10 \%$. For therapy, it is essential to stop the drug. To reduce the time required to normalize granulopoiesis from 1-2 weeks to a few days, the application of granulocyte growth factor (G-CSF) or granulocyte transfusion and the use of broad spectrum antibiotics to protect bacterial infections are recommended (3).

Severe congenital neutropenia or Kostmann's syndrome is characterized by severe neutropenia $\left(<500\right.$ cellsx $10^{6} \%$ litre), recurrent bacterial infections and failure in the maturation from promielocytes into mielocytes. At first it was classified as a recessive autosomal disease, but the underlying mutation has not been found yet (1). Oral problems include painful aphthous ulcers and gingivitis, and despite thorough dental care, children with congenital neutropenia tend to develop severe periodontal disease early in life. Although treatment with G-CSF has improved the control of bacterial infections, including gingivitis and peridontitis, many patients still experience periodontal disease, despite normal neutrophil counts (4).

Benign congenital neutropenia includes several benign 
disorders. They present scarce and generally mild mucocutaneous infections. Neutropenia usually becomes chronic although spontaneous remissions during childhood have been described. In any case, treatment is not needed (1). Several case reports have been published describing periodontitis as a manifestation of chronic neutropenia (5).

Cyclic neutropenia is characterized by a very severe neutropenia ( $<200$ cells $\times 10^{6} /$ litre) lasting from 3 to 6 days every 21 days, although in some cases the hematopoiesis cycles can vary from 2 to 5 weeks. It can be asymptomatic except in the neutropenic stage when aphthous ulcers, gingivitis, stomatitis and cellulitis appear (1).

\section{Neutrophil functional alterations}

Neutrophil dysfunctions are usually associated with hereditary intrinsic cell defects, although sometimes they are a consequence of extrinsic etiological factors such as prescription drugs, diabetes and certain infections.

Infections in patients with alterations in neutrophil function possess clinical characteristics that are conditioned by the kind of functional alteration.

In patients with leukocyte adhesion defects, the lesions have less pus and are formed by necrotic tissues with few inflammatory components.

By contrast, in the case of defects in the neutrophil migration the abscesses present abundant cells (neutrophils and macrophages), presumably due to the late arrival of neutrophils to the infected area.

In patients who suffer from a decrease in the neutrophil bactericide activity, as in the case of chronic granulomatous disease, abscesses are spread out, affect deep levels and have granulomas with or without purulent material. The skin and the affected tissues typically present pustules with a scarce inflammatory component.

Infections in patients who present alterations in the neutrophil function are often the consequence of common microorganisms in skin and mucous membranes such as Staphylococcus aureus, Pseudomonas, Candida spp. and Aspergillus spp. These patients, unlike neutropenic patients who suffer from bacteraemias and the dissemination of infections in deep tissues such as the bone ma- rrow, can commonly limit the infection to the lymphatic nodes and the liver, systemic involvement being infrequent (1).

In patients with lazy leukocyte syndrome stomatitis, gingivitis, and recurrent ulcerations have been reported (6).

Paediatric patients with neutrophil functional alterations such as chronic granulomatous disease and Chédiak-Higashi syndrome often suffer from mucous ulcerations, recurrent sinusitis, cervical lymphadenitis and candidiasis. Prepuberal periodontitis has been related to a leucocyte adhesion deficiency $(1,6)$ and generalized pre-puberal periodontitis, with neutrophil chemotaxis defects (1).

Dental management of patient with neutrophil alterations

Preventive measures and control of intraoral infectious processes, to minimize the necessity of surgical treatment, must be a constant factor in the dental treatment of these patients. Conventional preventive measures such as the use of topic fluoride, fissure sealants, dietary advice and the promotion of oral health, become especially important. It has been proven that the periodic removal (monthly) of plaque and calculus as well as the use of antiseptics (clorhexidine 0.2\%) and periodontal treatment in patients with cyclic neutropenia and Kostmann's syndrome help to mantain the periodontal insertion. Nevertheless, other patients with neutrophil dysfunction suffer unavoidably from periodontitis and dental mobility even with adequate care. As a consequence, periodontal surgery started to be considered only recently (1).

When oral surgical procedures are anticipated, a platelet count of $\geq 50,000$ cells $/ \mu \mathrm{L}$, absolute neutrophil count of $\geq 500$ cells $/ \mu \mathrm{L}$, and hemoglobin concentration of $\geq 7 \mathrm{~g} /$ $\mathrm{dL}$, are recommended to comfortably assure effective hemostasis and reduce the risk of postoperative bacterial infection (2). Other prophylactic measures to minimize the bacteraemia risk are: antiseptic rinse with clorhexidine prior to any dental treatment, antibiotic administration before and after surgery and the primary closing of the surgical wound (1).

Considerations in dental management of patients with neutrophil disorders are summarised in table 1.

\section{Considerations in dental management of patients with neutrophil disorders.}

\begin{tabular}{|l|l|}
\hline In all patients & In oral surgery \\
\hline - Application of topic fluoride and fissure sealants. & - Observe acceptable counts of platelets, neutrophils \\
- Dietary advice and promotion of oral health & and hemoglobin concentration. \\
- Antiseptic rinse with clorhexidine previous to any & - Antibiotic administration before and after surgery \\
dental treatment & - Primary closing of the surgical wound. \\
\hline
\end{tabular}

Table 1. Considerations in dental management of patients with neutrophil disorders. 


\section{Leukocytosis}

Leukocytosis is an increase in the number of leukocytes in the blood. We say that there is leukocytosis when the white blood cells count is higher than $10.000 / \mathrm{mm}^{3}$.

Leukocytosis, like neutropenia, is not a disease itself, but the sign of an underlying disorder of variable etiology. It is a common laboratory finding, often due to relatively benign conditions, as the normal reaction of bone marrow to infection or inflammation leads to an increase in the number of white blood cells, predominantly polymorphonuclear leukocytes and less mature cell forms (the "left shift"). Other causes, much less common but more serious, include primary bone marrow disorders. Physical stress and emotional stress can also elevate white blood cells counts. Drugs commonly associated with leukocytosis include corticosteroids, lithium and beta agonists. Increased eosinophil or basophil counts, resulting from a variety of infections, allergic reactions and other causes, can lead to leukocytosis in some patients (7).

Dental management of patient with leukocytosis

Dental treatment of patients with leukocytosis dont't need special considerations apart of ruling out oral pathology that could have caused leucocyte increase, and if found treat it as established. If we don't find any pathology that could increase the white cells count it is recommended to refer the patient to the specialist in order to rule out other diseases.

\section{HEMATOLOGIC MALIGNANCIES OF WHITE CELLS}

There are three main groups of hematologic malignancies: leukemia, lymphoma and plasma cell tumors. Leukemias are derived from hematopoyetic cells that proliferate initially in the bone marrow before spreading to peripheral blood, spleen, lymphatic nodes and, finally, to other tissues; they differ from lymphomas because these arise first in lymphatic nodes, however they can also spread to blood and bone marrow. Plasma cell tumors are lymphoid neoplastic proliferations of B cells.

\section{Leukemia}

Leukemia results from the proliferation of a clone of abnormal hematopoietic cells with impaired differentiation, regulation, and programmed cell death (apoptosis). Leukemic cell multiplication at the expense of normal hematopoietic cell lines causes marrow failure, depressed blood cell count (cytopenia), and death as a result of infection, bleeding, or both (8).

Leukemia is classified according to the primary hematopoietic cell affected (myeloid or lymphoid) and by the acute or chronic behavior $(8,9)$.

Chronic leukemia, with a less pronounced marrow failure, has an indolent course that usually lasts several years.
Acute leukemia is usually precipitous, with bone marrow failure and associated anemia, infection, and bleeding. Symptoms are generally flu-like with bone pain, joint pain, or both, caused by malignant marrow expansion. Thrombocytopenia is manifested by petechial skin and posterior palate hemorrhages and gingival bleeding. Acute myelogeous leukemia symptoms include fever, fatigue, pallor, mucosal bleeding, petechiae, and local infections; clinical manifestations of acute lymphocytic leukemia are similar to those of acute myelogeous leukemia, but with a high incidence of central nervous system disease (8).

Gingival hyperplasia secondary to infiltration of the gingival tissue with leukemia cells is thoroughly described in the literature (8-11). It is characterized by progressive enlargement of the interdental papillae as well as the marginal and attached gingival. In the condition's most pronounced form, the crowns of the teeth may be covered. Gingiva appear swollen, devoid of stippling and pale red to deep purple in colour (8). Gingival infiltration by leukemic cells will also predispose the patient with leukemia to bleeding (10). Gingival hiperplasia is more common in acute tan chronic leukemia $(8,10)$, nonetheless, the development of gingival infiltration is unpredictable in any individual patient. Generally, gingival hyperplasia resolves completely or at least partly with effective leukemia chemotherapy (10).

Gingival ulcerations may occur as a result of infection by normal oral flora in the setting of neutropenia (8).

\section{Lymphomas}

Lymphomas are a diverse group of neoplasms affecting the lymphoreticular system. Lymphomas have been traditionally divided into Hodgkin's disease and non Hodgkin's disease. Hodgkin's disease is characterized histologically by presence of multi-nucleated Reed-Sternberg cells. All other neoplasms of the lymphoid system are referred to as Non-Hodgkin's lymphoma and are derived predominantly from the cells of B-lymphocyte series (12). These diseases differ in a number of key clinical aspects (13).

Hodgkin lymphoma (HL) most frequently presents as cervical lymphadenopathy and rarely involves extranodal sites $(12,13)$. LH spreads in a predictable manner along lymphatic channels from one involved lymph node group to contiguous lymph nodes; because of this pattern of spread, limited HL is more amenable to cure with radiotherapy. The seldomly reported lesions include different locations: palate, tonsil, floor of mouth, buccal alveolar mucosa, buccal vestibule, and mandibular bone, with no one site accounting for a predominance of cases. The more commonly described clinical presentations are ulcerations and swellings (13).

Non-Hodgkin lymphoma (NHL) manifests up to $40 \%$ of the time at an extra nodal site. Moreover, 2-3\% of these 
extra nodal cases may arise primarily in the oral cavity and jaws (12). Nonetheless, NHL is the second most common oropharingeal malignancy after squamous cell carcinoma. Waldeyer's ring is most commonly involved, although any site may be affected (14). Oral lesions may appear as an erythematous, painless enlargement, often with surface ulceration secondary to trauma $(14,15)$, but rarely with deep oral ulceration (14). Jaw involvement by NHL is rare, but among jaw lesions, maxilla is more frequently involved than mandible (12). Radiographic findings may include diffuse bone destruction, and loss of the lamina dura. It is commonly associated with teeth loosening, and paresthesia, hardly ever being accompanied by "B symptoms": fever of unknown origin $\left(>38^{\circ} \mathrm{C}\right)$, unexplained weight loss $(>10 \%$ over 6 months), drenching night sweats, visceral pain and malaise $(14,15)$.

\section{Plasma cell neoplasia}

Plasma cell tumors are lymphoid neoplastic proliferations of B cells that may develop in a disseminated manner, affecting several bones (multiple myeloma) or less commonly as a solitary lesion at any interbone site (solitary bone plasmacytoma) or in soft tissues (extramedullary plasmacytoma).

In $12-15 \%$ of multiple myeloma (MM) cases, the onset of this process is determined by oral symptoms, being the most common toothache, oral or facial pain and deviations of the mandible. Bone lesions in MM are visible radiographically as well-defined, solitary or multiple radiolucent areas, without peripheral reinforcement, showing a tendency to converge and present images with septa or in "soap bubbles".

Solitary bone plasmacytoma rarely affects the oral cavity, but when it does, it shows a predilection for the retromolar area of the mandible. Its radiological appearance may have one of two patterns, as either an oval-shaped lytic image with destruction of the cortical bone, or as a hyperinsufflating lesion showing a convex bicortical bone.

Extramedullary plasmacytoma may be found in any part of the body having lymphoid tissue. In $90 \%$ of the cases this disease develops in the head and neck, showing a preference for the upper respiratory and digestive tracts. Clinically, they appear as a sessile or pediculated outgrowth, either circumscribed or infiltrating. There are no specific clinical manifestations as symptoms and they depend on the location of the tumor (16).

Dental management of patient with hematologic malignancies.

Patients in long-term remission can undergo dental treatment, while patients with advanced or relapsed disease with reserved prognosis should receive palliative or urgent treatment only. Dental treatment should be performed always after consultation with the specialist, as it can be modified by certain aspects of the therapy and of the disease prognosis (17).

It is very important to carry out a detailed history. This should include the diagnosis and status of the neoplasia, the nature and duration of previous and ongoing treatment, prognosis, comorbid medical conditions, current medications, past dental history, and prior history of oral and nonoral infections (14).

All patients with hematologic malignancies should undergo comprehensive oral and dental evaluation in which is important to:

- Evaluate for presence of petechiae, ecchymosis, gingival hemorrhage, tooth mobility, tooth migration, facial pain, and/or parestesia, indicative of local manifestations of hematologic malignancies.

- Evaluate for presence of dental/periodontal disease as a risk factor for development of osteonecrosis of the jaw (ONJ) and/or bacteriemia.

- Evaluate radiographs (panoramic and periapicals, as indicated) for evidence of osteolytic lesions (if detected, refer for further radiographic evaluation) and to determine potential risk for developing ONJ (17).

Dental treatment of these patients should be performed before starting the chemo/radiotherapy, treating any teeth with pulpal and periapical pathology, periodontal abscess, pericoronaritis, gross clinical caries and periodondontal disease. In the case of patients scheduled for autologous hematopoietic cell transplant, dental treatment should be avoided during the 3 days when stem cells are mobilized and harvested to minimize contamination secondary to iatrogenic transient bacteremia (14).

The main problems in dental treatment of patients with hematologic malignancies of white cells are:

1. Tendency to bleed:

- Intraoral bleeding is commonly observed, manifesting with petechiae and ecchymoses, and occasionally hematoma formation. These lesions do not require treatment, and large hematomas should not be excised due to risk of hemorrhage.

- For routine operative care where bleeding is not anticipated, treatment can generally be provided in even severely thrombocytopenic patients without the need for transfusions. Depending on the extent of surgery, coordination of platelet transfusion the day of the procedure may be indicated, although single tooth extractions can generally be managed by an experienced clinician with localized measures even with levels as low as 15,000 cells/mL (14).

2. Increased risk of infection:

Patients with compromised lymphocyte function or low neutrophil count due to their malignancy or secondary to myelosuppressive chemotherapy are prone to newly acquired infections and/or exacerbation or reactivation of latent infections $(14,18,19)$. In many cases the clinical 
presentation of oral infections may be atypical compared to what would normally be expected in a healthy patient population. Use of diagnostic tools, including cultures, cytological smears, and tissue biopsies, is critical in identifying pathogens and guiding appropriate therapies (14).

a) Odontogenic infections:

Oral prophylaxis, oral hygiene instruction and elimination of oral sources of infection before beginning cancer treatment, can significantly reduce the risk of infectious complications $(14,19)$. Odontogenic infections of pulpal and periodontal origin are frequently encountered and should be suspected in the presence of orofacial pain, large restorations, gross clinical caries, and periapical radiolucency. Dental radiographs must be obtained for any suspected odontogenic infection. Endodontically treated teeth with a radiographically adequate seal can become symptomatic during severe neutropenia, and while these may be initially managed with antibiotics, in most cases extraction is indicated. Similarly, chronic periodontal disease can become acutely exacerbated with or without the traditional clinical signs of inflammation and swelling. Treatment includes broad-spectrum antibiotics, chlorhexidine rinses, scaling and curettage, and extraction of hopeless teeth. Extractions should be performed as early as possible prior to beginning therapy to allow maximum healing. Prior to extractions or any other invasive procedures the platelet and absolute neutrophils counts must be reviewed and appropriate measures taken to minimize risk of complications (14).

b) Opportunistic infections:

- Viral infections: primary infection or reactivation of herpes family viruses is common in these patients, especially during intensive chemotherapy and in the context of advanced disease. Herpex simplex virus is the most common viral infection in these patients and typically presents as single or multiple painful ulcerative lesions that may involve any oral mucosal surface. Varicella-zoster virus (VZV) reactivation is less common. Treatments for both infections include acyclovir, valacyclovir or famciclovir, the latter appearing to have an advantage in preventing post-herpetic neuralgia following VZV infections $(14,19)$.

- Fungal infections: oropharingeal candidiasis is the most common fungal infection in cancer patients. Candidiasis can present as pseudomembranous (the most common), erythematous, hyperplastic, or angular cheilitis. Symptoms include generalized discomfort, dysgeusia, xerostomia, and burning. Initial episodes can be treated with topical azoles or nystatin for 7 to 14 days; in severe cases, systemic therapy should be considered with 100 to $200 \mathrm{mg}$ /day of fluconazole or itraconazole for 7 to 14 days or long-term for prophylaxis, especially in recurrent cases. In patients with nonhealing solitary ulcerations, deep fun- gal infections (aspergillus, zygomyces, and histoplasma) should be considered, the management of which requires aggressive therapy with intravenous azoles, amphotericin B, and the echinocandins $(14,19)$.

3. Risk of developing ONJ: in patients treated with radiotherapy or intravenous bisphosphonates there is a risk of developing osteonecrosis and it is recommended to follow the corresponding protocols.

4. Anemia

- Patients with severe anemia often complain of that they fatigue easily and may not be able to tolerate time-consuming dental treatment.

- The need for blood products and the appropriate venue for dental treatment should be discussed with the patient's oncologist, as these patients may require continuous monitoring of vital signs and blood counts perioperatively (17).

5. Corticosteroids treatment: patients receiving high-dose systemic corticosteroids may display evidence of secondary adrenal insufficiency. Adrenal crises secondary to insufficiency are rare in the dental setting, and steroid supplementation before nonsurgical dental procedures is not recommended. Steroid supplementation before oral surgery is usually recommended. The amount, duration, and venue for supplementation should be determined by both the dental specialist performing the procedure and the patient's oncologist (17).

6. Secondary malignancy: patients treated for hematologic malignancies are at high risk for relapse of primary disease as well as developing second hematologic and solid malignancies. Considering the increased risk of second primary cancer of the head and neck in the survivors of leukemia and lymphoma, and the fact that squamous cell carcinoma is the most common second primary solid malignancy after allogeneic hematopoyetic cell transplant, oral health care professionals play a critical role in the long-term surveillance of this patient population (14).

7. Specific considerations:

- Patients with renal dysfunction may require modified dosing intervals of medications (17).

- In patients with multiple myeloma, it is important to evaluate for presence of hard/soft tissue masses that could indicate deposition of plasma cells and/or lightchain associated amyloid, and biopsy if necessary (17).

- Patients with multiple myeloma and significant bone pain, especially in the back, may need frequent breaks and may require frequent repositioning during dental procedures (17).

- In patients undergoing orthodontic treatment, the removal of orthodontic appliances and delivery of retainers is recommended, as well as the postponement of orthodontic treatment until the patient has finished immunosuppressive therapy and the risk of hemato- 


\section{Considerations in dental management of patients with hematologic malignancies}

\section{Prior to dental treatment}

1. Patients in long-term remission can undergo dental treatment, while patients with advanced or relapsed disease with reserved prognosis should receive palliative or urgent treatment only.

2. Dental treatment should be performed always after consultation with the specialist

3. It is important to carry out a detailed history, a comprehensive oral and dental evaluation and a complete radiographic exam. 4. Dental treatment should be performed before starting the chemo/radiotherapy.

\section{During dental treatment}

1. Bleeding tendency

2. Increased risk of infection.

3. Risk of developing osteonecrosis of the jaw.

4. Anemia

5. Corticosteroids treatment.

6. Secondary malignancies.

7. Specific considerations.

Table 2. Considerations in dental management of patients with hematologic malignancies

logic relapse requiring further intervention is reduced $(20,21)$.

Considerations in dental treatment of patients with hematologic malignancies are summarized in table 2 .

\section{References}

1. Diz-Dios P, Ocampo-Hermida A, Fernandez-Feijoo J. Quantitative and functional neutrophil deficiencies. Med Oral. 2002;7:206-21.

2. Tewari S, Tewari S, Sharma RK, Abrol P, Sen R. Necrotizing stomatitis: a possible periodontal manifestation of deferiprone-induced agranulocytosis. Oral Surg Oral Med Oral Pathol Oral Radiol Endod. 2009; 108:e13-9.

3. Becker ST, Wiltfang J, Springer IN, Bartsch MS, Guenther R, Sherry E, et al. Life-threatening bite injury of the lower lip after use of metamizole. Oral Maxillofac Surg. 2008;12:39-41.

4. Carlsson G, Wahlin YB, Johansson A, Olsson A, Eriksson T, Claesson R, et al. Periodontal disease in patients from the original Kostmann family with severe congenital neutropenia. J Periodontol. 2006;77:744-51.

5. Zaromb A, Chamberlain D, Schoor R, Almas K, Blei F. Periodontitis as a manifestation of chronic benign neutropenia. J Periodontol. 2006 ;77:1921-6.

6. Van Dyke TE, Hoop GA. Neutrophil function and oral disease. Crit Rev Oral Biol Med. 1990;1:117-33.

7. Abramson N, Melton B. Leukocytosis: basics of clinical assessment. Am Fam Physician. 2000 1;62:2053-60.

8. McKenna SJ. Leukemia. Oral Surg Oral Med Oral Pathol Oral Radiol Endod. 2000; 89:137-9.

9. Demirer S, Ozdemir H, Sencan M, Marakoglu I. Gingival hyperplasia as an early diagnostic oral manifestation in acute monocytic leukemia: a case report. Eur J Dent. 2007;1:111-4.

10. Cooper CL, Loewen R, Shore T. Gingival hyperplasia complicating acute myelomonocytic leukemia. J Can Dent Assoc. 2000;66:78-9.

11. Koulocheris P, Metzger MC, Kesting MR, Hohlweg-Majert B. Life-threatening complications associated with acute monocytic leukaemia after dental treatment. Aust Dent J. 2009;54:45-8.

12. Kini R, Saha A, Naik V. Diffuse large B-cell lymphoma of mandible: a case report. Med Oral Patol Oral Cir Bucal. 2009;14:e421-4.

13. Whitt JC, Dunlap CL, Martin KF. Oral Hodgkin lymphoma: a wolf in wolf's clothing. Oral Surg Oral Med Oral Pathol Oral Radiol Endod. 2007; 104:e45-51.

14. Mawardi H, Cutler C, Treister N. Medical management update:
Non-Hodgkin lymphoma. Oral Surg Oral Med Oral Pathol Oral Radiol Endod. 2009;107:e19-33.

15. Van der Waal RI, Huijgens PC, van der Valk P, van der Waal I. Characteristics of 40 primary extranodal non-Hodgkin lymphomas of the oral cavity in perspective of the new WHO classification and the International Prognostic Index. Int J Oral Maxillofac Surg. 2005;34:391-5. 16. Seoane J, Aguirre-Urizar JM, Esparza-Gómez G, Suárez-Cunqueiro M, Campos-Trapero J, Pomareda M. The spectrum of plasma cell neoplasia in oral pathology. Med Oral. 2003;8:269-80.

17. Stoopler ET, Vogl DT, Stadtmauer EA. Medical management update: multiple myeloma. Oral Surg Oral Med Oral Pathol Oral Radiol Endod. 2007;103:599-609.

18. Raut A, Huryn JM, Hwang FR, Zlotolow IM. Sequelae and complications related to dental extractions in patients with hematologic malignancies and the impact on medical outcome. Oral Surg Oral Med Oral Pathol Oral Radiol Endod. 2001;92:49-55.

19. Heimdahl A. Prevention and management of oral infections in cancer patients. Support Care Cancer. 1999;7:224-8.

20. Sheller B, Williams B. Orthodontic management of patients with hematologic malignancies. Am J Orthod Dentofacial Orthop. 1996;109:575-80.

21. Isaac AM, Tholouli E. Orthodontic treatment for a patient who developed acute myeloid leukemia. Am J Orthod Dentofacial Orthop. 2008;134:684-8. 